\title{
Contribution of the Primate Superior Colliculus to Inhibition of Return
}

\author{
Michael C. Dorris ${ }^{1}$, Raymond M. Klein ${ }^{2}$, Stefan Everling1, \\ and Douglas P. Munoz
}

\begin{abstract}
The phenomenon of inhibition of return (IOR) has generated considerable interest in cognitive neuroscience because of its putative functional role in visual search, that of placing inhibitory tags on objects that have been recently inspected so as to direct further search to novel items. Many behavioral parameters of this phenomenon have been clearly delineated, and based on indirect but converging evidence, the widely held consensus is that the midbrain superior colliculus (SC) is involved in the generation of IOR. We had previously trained monkeys on a saccadic IOR task and showed that they displayed IOR in a manner similar to that observed in humans. Here we recorded the activity of single neurons in the superficial and intermediate layers of the SC while the monkeys performed this IOR task. We found that when the target was presented at a previously cued location,
\end{abstract}

\section{INTRODUCTION}

The sudden presentation of an uninformative visual cue initially enhances responding but is followed by a period of increased reaction times to stimuli presented at the previously cued location. Being interpreted as the inhibition of attentional resources from returning to previously attended locations, the delayed responding was labeled "inhibition of return" (IOR) (Posner \& Cohen, 1984; Posner, Rafal, Choate, \& Vaughan, 1985). This phenomenon is intriguing because IOR may have a functional role in making visual search more efficient (Klein \& MacInnes, 1999; Danziger, Kingstone, \& Snyder, 1998; Taylor \& Klein, 1998; Posner \& Cohen, 1984; Posner et al., 1985), a view that is bolstered by studies that show that up to five previously cued objects in a visual scene are "tagged" with inhibition (Snyder \& Kingstone, 2000) and that IOR remains despite movement of a cued object (Tipper, Weaver, Jerreat, \& Burak, 1994) and following shifts of gaze (Maylor \& Hockey, 1985).

The primate superior colliculus (SC) is a critical node in the visual orienting pathway (Munoz, Dorris, Pare, \&

\footnotetext{
${ }^{1}$ Queen's University, Ontario, Canada, ${ }^{2}$ Dalhousie University, Nova Scotia, Canada
}

the stimulus-related response was attenuated and the magnitude of this response was correlated with subsequent saccadic reaction times. Surprisingly, this observed attenuation of activity during IOR was not caused by active inhibition of these neurons because (a) they were, in fact, more active following the presentation of the cue in their response field, and (b) when we repeated the same experiment while using the saccadic response time induced by electrical microstimulation of the SC to judge the level of excitability of the SC circuitry during the IOR task, we found faster saccades were elicited from the cued location. Our findings demonstrate that the primate SC participates in the expression of IOR; however, the SC is not the site of the inhibition. Instead, the reduced activity in the SC reflects a signal reduction that has taken place upstream.

Everling, 2000), and various lines of evidence have suggested that it is involved in the generation of IOR (Rafal, Calabresi, Brennan, \& Sciolto, 1989). IOR is reduced or absent in patients with lesions to the SC (Sapir, Soroker, Berger, \& Henik, 1999; Posner \& Cohen, 1984; Posner et al., 1985) and has been observed in the hemianopic field of a patient with cortical damage (Danziger, Fendrich, \& Rafal, 1997) and in newborns (Simion, Valenza, Umilta, \& Dalla Barba, 1995) for whom the SC but not the cortex is fully developed. Under the assumption of greater temporal than nasal hemifield retinotectal projections (but see Perry \& Cowey, 1984), further evidence for SC mediation has been attributed to the finding that IOR is larger in the temporal than nasal hemifield (Rafal et al., 1989). Other evidence, however, points to a role for cortical areas in the coding of IOR. For example, IOR affects manual response times whereas the SC has been traditionally viewed as an oculomotor structure. Furthermore, the inhibition of responses is maintained if the eyes (Maylor \& Hockey, 1985) or tagged objects (Tipper et al., 1994) move, suggesting IOR is coded in a coordinate frame outside the oculocentric coordinate frame used by the SC.

Our previous work using a cue-saccade task explored this phenomenon behaviorally in monkeys 
and demonstrated their suitability as an animal model (Dorris, Taylor, Klein, \& Munoz, 1999). Using this same task, our goal was foremost to search for evidence of $\mathrm{SC}$ involvement in IOR using the direct technique of extracellular microelectrode recording. If the SC was indeed involved, then we expected to be able to use this preparation to expand our knowledge of the nature of IOR. We found that the initial stimulusrelated response of SC superficial and intermediate layer neurons to the presentation of saccadic targets is strongly attenuated if a cue had been presented previously at the same location. In addition, the level of this activity is well correlated to ensuing reaction times. However, neuronal activity was enhanced during the period between the initial cue and the presentation of the saccadic target. We directly tested the excitability of SC circuitry during IOR by measuring saccadic reaction times elicited after the application of electrical current to the SC during the IOR task. Our results support the hypothesis that the SC is involved in the generation of oculomotor IOR, but they also suggest that the SC is not the site of inhibition; rather it receives reduced inputs from upstream structures in response to previously cued targets.

\section{RESULTS}

We recorded the activity of single neurons in the SC while monkeys performed a saccadic IOR paradigm (Figure 1A). The initial uninformative visual stimulus (S1) was presented at either the "same" or "opposite" location as the upcoming saccadic target (T2). The monkey was required to remain fixated on a central fixation point until it disappeared, which coincided with $\mathrm{T} 2$ appearance. The time between S1 appearance and T2 appearance (stimulus onset asynchrony [SOA]) was randomly varied between 200 or 1100 msec.

The activity of an SC neuron, which displayed both an initial visual response time-locked to the presentation of the stimulus, and a second motor response time-locked to saccades directed to the target in its response field, is contrasted during the same and opposite conditions (Figure 1B; note that only $50 \%$ of trials in which the target appeared in the response field of the neuron are
Figure 1. Neural activity during the inhibition of return (IOR) paradigm. (A) Schematic of the visual display showing the relative locations of the central fixation point (cross) and $\mathrm{S} 1$ and $\mathrm{T} 2$ locations (circles). The arrow represents a saccade directed into the response field of the neuron (dashed circle). (B) The activity of a single visual-motor neuron is segregated off-line into same (dotted spike density traces) and opposite (solid spike density traces) trials during the 200-msec SOA IOR paradigm. All traces are aligned on the time from T2 appearance in the neuron's response field, and time -200 msec represents the time of S1 presentation as depicted by the schematic at the bottom of the figure. Each raster (top panels) represents an action potential from the neuron and each line of rasters represents a single trial. The gray area $(70-120 \mathrm{msec}$ after

T2 presentation) represents the period during which the peak neuronal activity was sampled for subsequent analysis. (C) The peak T2 stimulus-related activity is plotted for both the opposite and same conditions for each of the neurons $(n=48)$. Empty circles and filled squares represent the activity from superficial layer visual neurons and intermediate layer visual-motor neurons, respectively. There was no significant difference between superficial visual neurons $(n=19)$, which displayed stimulus-related responses only, and intermediate layer visual-motor neurons $(n=29)$, which displayed both stimulus- and saccade-related responses, with respect to this analysis $(p=.35$, $t$ test). The majority of the data points fall above the line of unity (slope $=1$ ) meaning these neurons are more active during this sampling period for opposite than same trials. (D) For the same blocks of trials in which the neuronal activity was sampled in (C) we plotted the corresponding mean SRTs. For the majority of recorded neurons, the corresponding behavior displayed IOR being faster for the opposite than the same condition.

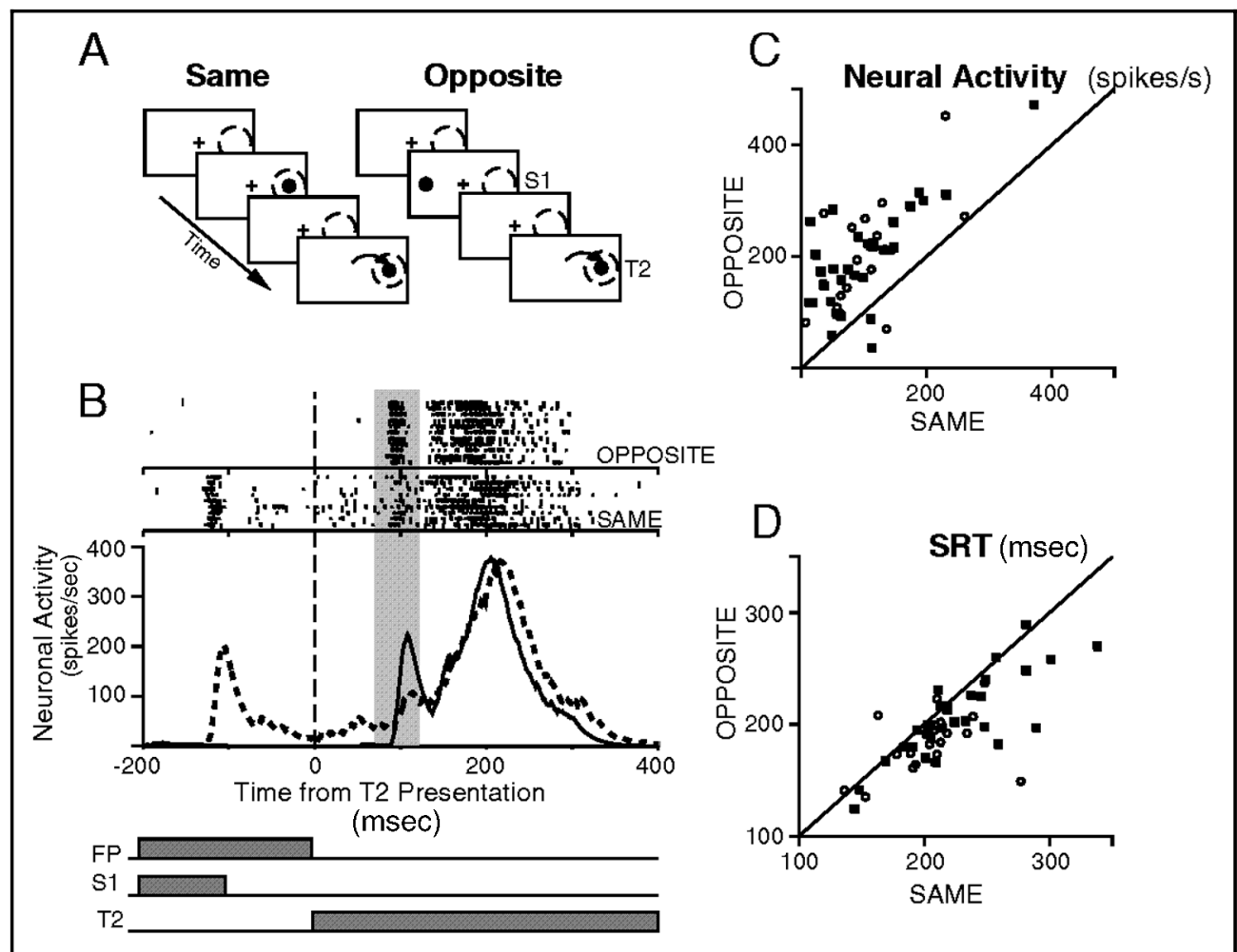


illustrated). During the "opposite" condition, S1 (shown here presented before target appearance with a 200-msec SOA) elicited no response from this neuron when its location was opposite to the neuron's response field. Naturally, during the "same" condition, S1 elicited a stimulus-related response when presented in the neuron's response field.

A striking difference between the activity patterns in the same and opposite conditions is seen in the stimulus-related response associated with the presentation of T2 in the neuron's response field (i.e., difference between peaks during the gray epoch in Figure 1B). Although the identical T2 stimulus was presented at the identical location, the magnitude of the stimulus-related response was dependent upon the previous S1 location, being attenuated when $\mathrm{T} 2$ was presented at the same location as S1. The T2 saccade-related response, however, was of similar magnitude for both the same and opposite conditions, the only difference being that this response was delayed in the same condition in keeping with the longer SRTs of IOR. When the neuronal activity was aligned on saccade onset, there was no significant difference in the magnitude of the saccade-related activity for the same versus the opposite condition for our sample of neurons (not shown, $p=.69$, paired $t$ test, $n=29$ neurons with saccade-related responses). Therefore, it does not appear that under these conditions, IOR is due to a specific diminishment in the magnitude of the motor command at the level of the SC.

Another difference in the neural activity recorded in the same and opposite conditions was seen during the interval between S1 and T2 presentation in which the discharge rate was higher for the same condition (Figure 1B). That is, one aftermath of a peripheral stimulus is a general increase in the discharge rate of neurons activated by the stimulus. This strongly suggests that IOR is not caused by active inhibition of recently stimulated SC neurons because such an inhibition would, other things being equal, entail a lower level of excitability. If recently stimulated SC neurons are not actively inhibited, then their reduced responsivity to subsequent stimuli might be attributed to a reduction in the magnitude of T2 stimulus-related inputs onto these neurons.

The majority of neurons studied (45/48) had a greater T2 stimulus-related response for the opposite condition than for the same condition for the 200-msec SOA (Figure 1C). While we recorded the neuronal activity for each experimental session, we also measured the corresponding behavior (Figure 1D). Higher T2 stimulus-related activity during the opposite condition (Figure 1C) corresponded with shorter SRTs (Figure 1D) for the majority of blocks of trials (41/48). It has been demonstrated that this behavioral IOR effect is not caused by a sensory-masking mechanism because IOR displays interocular transfer (Tassinari \& Berlucchi, 1993), is coded in environmental/object-centered coor-

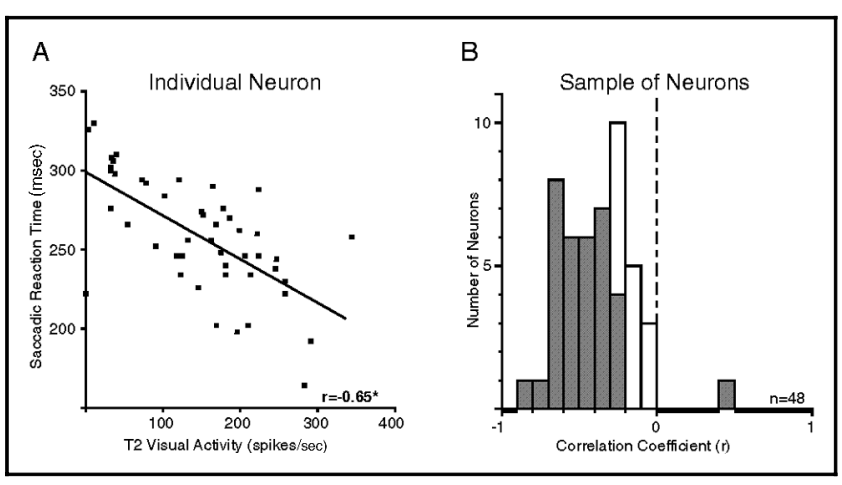

Figure 2. Trial-by-trial correlation between the magnitude of the T2 stimulus-related activity and SRT. (A) Data from one neuron in which the final T2 saccade was directed into the neuron's response field is shown. Empty circles and filled squares represent opposite and same condition trials, respectively. Each data point represents a single trial in which the magnitude of the T2 stimulus-related activity is plotted against the corresponding SRT. There is a significant negative correlation between these two variables $(p<.01)$. (B) The correlation coefficients for the analysis in (A) are plotted for the sample of 48 neurons. The gray bars represent the neurons with statistically significant correlations $(p<.05)$ between neuronal activity and SRT.

dinates (Tipper et al., 1994), and at shorter SOAs than used in this study facilitation (rather than inhibition) occurs at the same location (Briand, Larrison, \& Sereno, 2000; Fecteau and Munoz, unpublished observations). On average, for the 200-msec SOA, SRTs were $20 \mathrm{msec}$ slower for targets at the previously cued (same) than at the uncued (opposite) location $(p<.0001$, paired $t$ test) and the magnitude of the T2 stimulus-related response showed a corresponding decrease of 97 spikes/sec at the cued (same) location $(p<.0001$, paired $t$ test).

When the SOA was increased to $1100 \mathrm{msec}$ (not shown) the difference between the opposite and same conditions was reduced, being six spikes/sec for the T2 stimulus-related response ( $p=.20$, paired $t$ test) and $-2 \mathrm{msec}$ for SRT ( $p=.26$, paired $t$ test). Although the correspondence between neuronal activity and SRT was maintained, the loss of the behavioral IOR effect at the longer SOA differs from our previous study in which we observed IOR in these same three monkeys for both 650- and 1150-msec SOAs (Dorris et al., 1999). A recent study showing that the inhibition can disappear with practice (Weaver, Lupianez, \& Watson, 1998) suggests a possible explanation for the absence of IOR at $1100 \mathrm{msec}$, as these monkeys are probably the most heavily practiced subjects since the discovery of IOR.

We have shown a general relationship between the magnitude of the T2 stimulus-related response and SRT across separate sessions of neuronal recording. To address the issue whether this relationship is of sufficient strength to be a reliable predictor of SRT on a trial to trial basis, we measured the peak discharge $70-120 \mathrm{msec}$ after T2 appearance (gray bar in Figure 1B) in each neuron's response field and plotted it against the corresponding SRT for each trial (Figure 2A). A significant 
negative correlation is shown for a representative visualmotor neuron meaning that low T2 stimulus-related activity was predictive of long SRTs and high T2 stimulus-related activity was predictive of short SRTs. There was a negative correlation between $\mathrm{T} 2$ stimulus-related activity and SRT for nearly all of our neurons (47/48) with the majority of these correlations being statistically significant ( $p<.05 ; 33$ negative, 1 positive). Overall, the mean correlation coefficient for the sample of neurons was -.39 , which differed significantly from zero $(p<.0001$, paired $t$ test assuming unequal variances). Therefore, the T2 stimulus-related activity of SC neurons is related to IOR on both a gross level and on a trial by trial basis. This finding represents the most direct evidence, thus far, that the SC is involved in the manifestation of IOR.

Although our results support the hypothesis that the $\mathrm{SC}$ is in the pathway subserving IOR, we have not determined whether the SC is the site of inhibition or whether inhibition occurs elsewhere and the SC receives reduced T2 stimulus-related inputs. We observed that there was increased activity during the SOA period in the same condition compared to the opposite condition (Figures $1 \mathrm{~B}$ and $3 \mathrm{~A}$ ). This suggests that these neurons are not under active inhibition after presentation of the initial cue and lends support to the latter view. To directly distinguish between these two possibilities, we performed a stimulation IOR experiment (Figure 3). This was identical to the previous paradigm except that on $25 \%$ of the trials we evoked an eye movement with electrical microstimulation of the SC intermediate layers in lieu of $\mathrm{T} 2$ presentation (Figure 3B). The time to initiate an electrically induced saccade should depend on the level of preexisting neural excitability (Munoz et al., 2000; Stanford, Freedman, \& Sparks, 1996; Hikosaka \& Wurtz, 1985). If SC neurons are actively inhibited during the same condition, then more time should be required to reach saccadic threshold resulting in longer SRTs than those elicited in the opposite condition (i.e., IOR pattern of SRTs would remain for electrically induced saccades). If, however, these neurons are not actively inhibited during the same condition the IOR pattern seen in SRTs (i.e., shorter SRTs in the same condition than in the opposite condition; Figure 3B) should be absent, or because our cell recordings show an increased level of activity (Figures $1 \mathrm{~B}$ and $3 \mathrm{~A}$ ), the IOR pattern could be reversed.

The results from the stimulation IOR experiment are shown in Figure 3C. As already demonstrated, SRTs show a significant IOR effect on the nonstimulated trials during the 200-msec SOA (empty bars; paired $t$ test, $p<.005)$. However, when saccades were elicited by electrical stimulation after a 200-msec SOA, there was a reversal of the IOR effect with the same condition being significantly faster than the opposite condition (filled bars; paired $t$ test, $p<.01$ ). It is important to note that this reversal of the IOR effect was not due to facilitation observed in some IOR experiments with extremely short SOAs. In the nonhuman primate, facilitation is only observed with an SOA of $50 \mathrm{msec}$; when the SOA was 80 or more milliseconds then only inhibition is observed (Fecteau and Munoz, unpublished observations). During the 1100-msec SOA, SRTs on the nonstimulated trials did not reveal a significant difference
Figure 3. Stimulation IOR paradigm. (A) A saccade is hypothesized to be initiated after the neuronal activity surpasses a threshold level. IOR is associated with delayed threshold crossing in the same condition (dotted traces) compared to the opposite condition (solid traces). (B) During the stimulation IOR paradigm, electrical stimulation (Stim) is applied instead of T2 presentation. Based on our neuronal recording data, which showed that the baseline activity was higher during the same condition at the time of $\mathrm{T} 2$ presentation, we hypothesized that these neurons are not actively inhibited and will show a reversed IOR effect with electrical stimulation.

(C) Histograms of mean results from eight stimulation sites. Control behavioral SRTs are shown with empty bars and stimulation-elicited reaction times are shown with filled bars. These results suggest that in the same condition, the SC is not actively inhibited but instead is receiving reduced input regarding the presentation of $\mathrm{T} 2$.

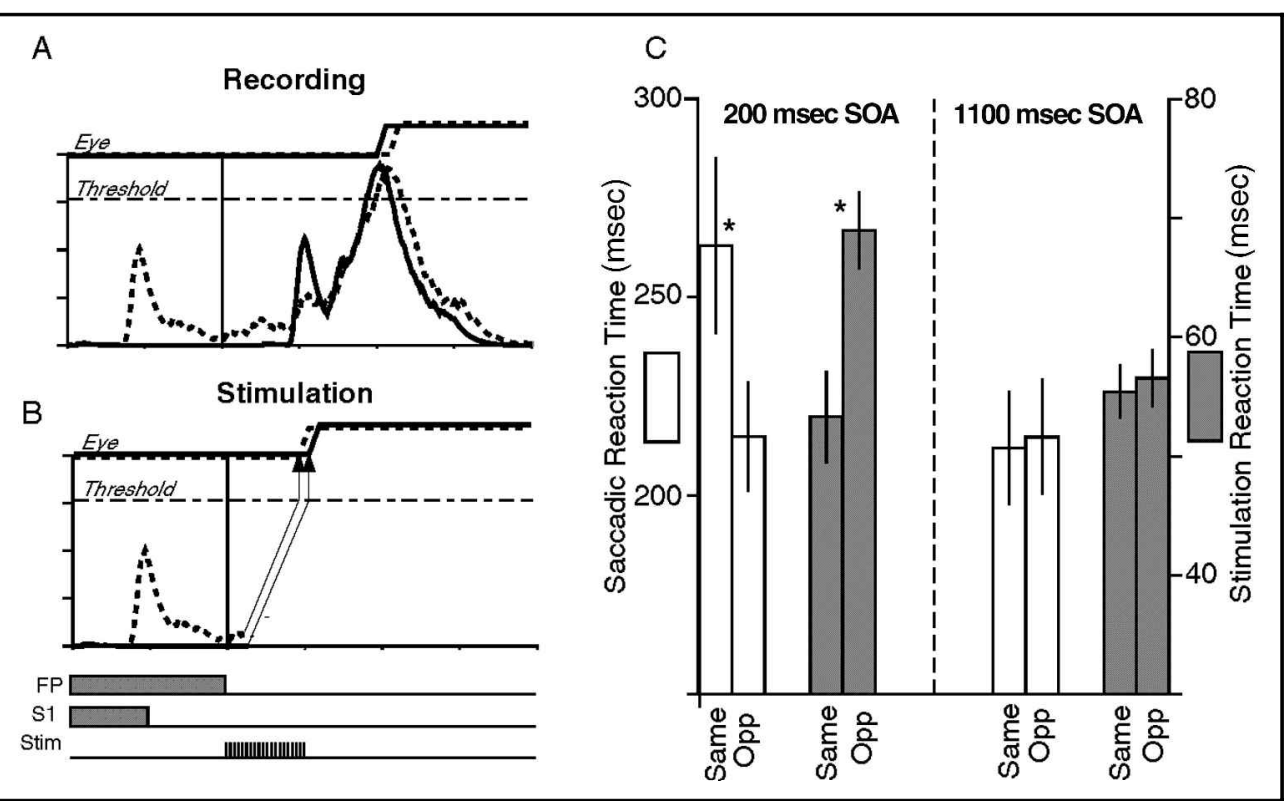


(paired $t$ test, $p>.05$ ) nor was there a significant latency difference on stimulated trials (paired $t$ test, $p>.05)$. The results of this experiment, in conjunction with the observation that SC neurons show a higher level of activity during the SOA period for same trials as opposed to opposite trials (Figure 1B), are inconsistent with the view that the SC is the site of inhibition in IOR. Rather, they support the hypothesis that the T2 stimulus-related response is attenuated upstream of the SC.

\section{DISCUSSION}

The use of nonhuman primates has proven to be a powerful tool in identifying the neural substrates and mechanisms underlying attention (Robinson, Bowman, \& Kertzman, 1995; Moran \& Desimone, 1985), visual search (Schall, 1995; Chelazzi, Miller, Duncan, \& Desimone, 1993), and response preparation (Dorris \& Munoz, 1998). It has been surmised that the phenomenon of IOR has properties common to all of these cognitive processes. The present work shows that IOR is not limited to humans but may be a process common to a number of species for optimizing the extraction of visual information from complex environmental surroundings. By showing strong correlations between neuronal activity and IOR behavior across experimental sessions (Figure 1C and D) and on a trial by trial basis within a single session (Figure 2) this study also provides the most direct evidence that the SC is involved in the manifestation of oculomotor IOR.

Nevertheless, whereas the SC may be involved in the manifestation of IOR, our results do not support the idea that the SC is itself inhibited when IOR is present. First, we found that the activity level of SC neurons during the SOA period is actually higher at a previously stimulated location (same condition) as compared to the location on the opposite side (opposite condition) (Figure 1B). Second, we found that saccades elicited artificially using electrical microstimulation of the SC saccadic circuitry were initiated more rapidly when the electrical stimulation was presented to the same location as the prior visual stimulus as compared to the opposite location. This reversal of the IOR effect combined with the heightened activity we observed in the SC following a stimulus (Figure 3) is not consistent with the idea that IOR is implemented as direct inhibition of the SC circuitry. Previous evidence is compatible with the view that inhibition occurs in sites upstream of the SC during IOR. First, IOR has been measured using both oculomotor and manual responses, whereas the SC has been traditionally viewed as an orienting structure of the visual axis (Munoz et al., 2000; Sparks \& HartwichYoung, 1989), although recent work has suggested a possible role for the SC in the control of arm movements (Stuphorn, Hoffmann, \& Miller, 1999). Second, IOR, at least when measured with manual responses, is coded both in environmental and object-centered coordinates (Tipper et al., 1994) whereas the SC uses an oculocentric code (Sparks \& Hartwich-Young, 1989).

Previously, Robinson and colleagues observed attenuation of stimulus-related responses to targets presented at previously cued locations in both the SC (Robinson \& Kertzman, 1995) and posterior parietal cortex (Robinson et al., 1995). These studies are notable because they employed very short SOAs (as low as $50 \mathrm{msec}$ ) and attenuation of stimulus-related activity was still observed, suggesting that attenuation begins almost immediately after the initial cue. These studies differ from ours in two important respects. First, even though the monkeys made manual rather than saccadic responses, attenuation still occurred in the SC. Second they employed a paradigm in which the cue was informative, that is it predicted the upcoming target location with $80 \%$ validity. Indeed, even while manual RTs were faster to targets appearing at the cued as compared to the uncued location there was a decrease in the stimulus-related responses elicited by cued targets in both the SC and parietal cortex. It appears, therefore, as though the attenuation of stimulus-related responses may be automatic because it occurs independently of whether an eye or hand response is required or whether the validity of the cues elicits reduced or increased reaction times. Finally, attenuation of cortical responses has been observed using an event-related potential IOR task (McDonald, Ward, Kiehl, 1999) and in response to repeated stimuli using a nonreaction time, delayed match to sample tasks in both the posterior parietal cortex (Steinmetz \& Constantinidis, 1995; Steinmetz, Connor, Constantinidis, \& McLaughlin, 1994) and the inferior temporal cortex (Miller, Gochin, \& Gross, 1991), suggesting that this is a ubiquitous response property throughout a variety of brain areas.

Since its discovery, scientists have been interested in "what is inhibited in inhibition of return" (ReuterLorenz, Jha, \& Rosenquist, 1996). Does IOR attenuate sensory/perceptual levels of analysis? Does it attenuate the return of attention (as implied by its name)? Or does it hinder nonattentional spatial responding? The attenuation of the stimulus-related response in the SC that we have reported here is compatible with an account that claims that IOR involves inhibition of the sensory/ perceptual analysis of stimuli presented at previously cued locations. Whereas this converges with studies showing poorer discriminability at previously cued locations (e.g., Pratt \& Castel 2001; Cheal \& Chastain 1999) it would need to be reconciled with studies showing that IOR does not delay a target's perceptual arrival time (for a review, see Klein, Schmidt, \& Muller, 1998). This reduced stimulus-related response is also compatible with an attentional account of IOR because this neural response is known to be influenced by and may partially code attentional processing (Wurtz \& Mohler, 1976). Finally, although there is now convincing evidence for a 
motoric component to IOR (Ivanoff \& Klein, 2001; Taylor \& Klein, 2000), we saw no evidence here that the magnitude of the motor burst in the SC was affected by IOR. On the contrary, there was greater activity in the cue-target interval in the same condition. This is perplexing because, in studies of motor preparation (Dorris, Pare, \& Munoz, 1997; Dorris \& Munoz, 1998) similar increases in pretarget activity were associated with shorter, not longer, reaction times.

It has been suggested that facilitation effects due to reflexive orienting of attention and the inhibitory effects of IOR may be independent effects that occur simultaneously but whose magnitudes follow different time courses (Klein, 2000; Ro \& Rafal, 1999; Tipper et al., 1997). This is bolstered by our observation of attenuation of stimulus-related responses with a 200-msec SOA and that of Robinson and Kertzman (1995) with a 50-msec SOA that suggest that this inhibition occurs as soon as the cue is presented. Simultaneously we observed increased activity during our shortest SOA period of $200 \mathrm{msec}$ that may be related to faster orienting at short SOAs. Recent studies in nonhuman primates have begun to vary SOAs systematically during a block of trials to reveal early facilitation $(\mathrm{SOA}=50 \mathrm{msec}$ ) and longer inhibition (SOA > $80 \mathrm{msec}$ ) (Fecteau and Munoz, unpublished observations). It will be important to determine how these excitatory and inhibitory processes evolve and are combined at the level of the SC.

We suggest that the posterior parietal cortex is a possible candidate for a cortical site that works in concert with the SC in generating IOR. This structure is intimately involved in attentional processing (Colby \& Goldberg, 1999) and the intention to make both saccadic and arm movements (Snyder, Batista, \& Andersen, 1997). Furthermore, visual space is constantly updated within this structure (Duhamel, Colby, \& Goldberg, 1992), which may provide a mechanism whereby inhibition can persist through movement of the eyes or the tagged object (Tipper et al., 1994; Maylor \& Hockey, 1985). Once an object or location has been tagged with inhibition, this information can be forwarded via direct corticotectal projections from the posterior parietal cortex (Paré \& Wurtz, 1997; Fries, 1984) so the SC can generate the appropriate saccadic behavior.

\section{METHODS}

\section{Subjects}

In three male rhesus monkeys (Macaca mulatta), we used standard techniques to record the extracellular activity of single neurons, to measure eye movements, and to convolve spike trains (as described previously; see Dorris \& Munoz, 1998). All procedures were approved by the Queen's University Animal Care Committee and complied with the guidelines of the Canadian Council on Animal Care.

\section{Task Procedures}

The monkeys were trained to perform a saccadic IOR paradigm (Figure 1A). Each trial was preceded by a 1000-msec intertrial interval. All stimuli were backprojected red-light-emitting diodes with a luminance of $2.0 \mathrm{~cd} / \mathrm{m}^{2}$. Each trial began with the presentation of a central fixation point (FP), which the monkey was required to fixate within $500 \mathrm{msec}$. After $500 \mathrm{msec}$ of fixation, an irrelevant eccentric stimulus (S1, uninformative peripheral cue) was flashed for $100 \mathrm{msec}$. The monkey was required to maintain fixation upon the FP and not respond to S1. The FP was extinguished after a pseudorandom period of 200 or $1100 \mathrm{msec}$ after the appearance of $\mathrm{S} 1$ (the interval referred to as stimulus onset asynchrony [SOA]), and simultaneously a target stimulus (T2) was presented. The monkey's task was to initiate a saccade to the target within $500 \mathrm{msec}$ of its appearance and then maintain fixation upon it for $300 \mathrm{msec}$. Although the cues and targets were identical, the monkeys were trained only to move their eyes to peripheral stimuli in the absence of the FP. If the monkey performed a trial correctly it received a liquid reward. If, however, at any time the monkey did not maintain fixation within the computer-controlled window around the FP or T2 (usually $3^{\circ} \times 3^{\circ}$ ), or did not meet the time constraints dictated by the paradigm, the trial was aborted and the monkey did not receive the liquid reward. The monkey worked to satiation and additional water and fruit were given as necessary. The locations of S1 and T2 were randomized between the center of a neuron's response field (as determined by the location in the visual field that elicited the most vigorous stimulus-related response) and the position opposite the neuron's response field at the same eccentricity but on the opposite side of the horizontal and vertical meridians. S1 was unpredictive of the upcoming T2 location. Each neuron was tested in a block that consisted of between 10 and 20 successfully completed trials of each of the eight conditions (2 S1 locations, 2 T2 locations, 2 SOAs). We restricted our analysis to trials in which the saccades ended within $3^{\circ}$ of the final saccadic target and with SRTs between 70 and $500 \mathrm{msec}$.

\section{Neuron Classification}

We analyzed the discharge of a subset of SC visual and visual-motor neurons recorded from the superficial and intermediate layers of the SC, respectively. To be included in our analysis, neurons were required to display a transient burst of activity that was time-locked to the presentation of S1 in the center of the neuron's response field that began $<100$ msec after S1 presentation and peaked with a discharge rate of at least 50 spikes/sec above baseline activity. The magnitude of the visual activity of both the visual and visual-motor 
neurons did not differ significantly during our analysis and are displayed separately only in Figure $1 C$ and D and are collapsed together for the remainder of the article.

\section{Stimulation Experiment}

A stimulation IOR paradigm was performed at eight sites in three SC of two monkeys. After recording was completed from an isolated visual-motor neuron in the intermediate layers of the SC, the preamplifier leads attached to the electrode were replaced with leads from a stimulator. Electrical stimulation (0.3-msec biphasic pulses for 100-msec duration at $200 \mathrm{~Hz}$ ) was applied during fixation of the FP to ensure that the electrically driven saccade was the same vector as that indicated by neuronal activity. The stimulation IOR paradigm was identical to the previously described paradigm except that on $25 \%$ of the trials electrical stimulation was applied either 200 or $1100 \mathrm{msec}$ after S1 appearance through the electrode in lieu of the presentation of T2 in the response field of the stimulation site. Before a block of trials was run, the current was adjusted such that it elicited a saccade on almost all trials yet allowed some variability in the reaction time of the evoked saccade. This resulted in a range of site stimulus intensities between 18 and $38 \mu \mathrm{A}$.

\section{Acknowledgments}

We thank A. Lablans and D. Hamburger for technical assistance. This work was supported by the McDonnell-Pew Cognitive Neuroscience Foundation. M. C. Dorris was supported by a Queen's University Graduate Fellowship and an Ontario Graduate Science and Technology Scholarship. D. P. Munoz holds a Canada Research Chair in Neuroscience.

Reprint requests should be sent to Dr. D. P. Munoz, Department of Physiology, Queen's University, Kingston, Ontario, Canada, K7L 3N6, or via e-mail: doug@eyeml.queensu.ca.

\section{REFERENCES}

Briand, K. A., Larrison, A. L., \& Sereno, A. B. (2000) Inhibition of return in manual and saccadic response systems. Perception and Psychophysics, 62, 1512-1524.

Cheal, M., \& Chastain, G. (1999). Inhibition of return: Support for generality of the phenomenon. Journal of General Psychology, 26, 375-390.

Chelazzi, L., Miller, E. K., Duncan, J., \& Desimone, R. (1993). A neural basis for visual search in inferior temporal cortex. Nature, 93, 345-347.

Colby, C. L., \& Goldberg, M. E. (1999). Space and attention in parietal cortex. Annual Review of Neuroscience, 22, 319-349.

Danziger, S., Fendrich, R., \& Rafal, R. (1997). Inhibitory tagging of locations in the blind field of hemianopic patients. Consciousness and Cognition, 6, 291-307.

Danziger, S., Kingstone, A., \& Snyder, J. J. (1998). Inhibition of return to successively stimulated locations in a sequential visual search paradigm. Journal of Experimental Psychology: Human Perception and Performance, 24, $1467-1475$.
Dorris, M. C., \& Munoz, D. P. (1998). Saccadic probability influences motor preparation signals and time to saccadic initiation. Journal of Neuroscience, 98, 7015-7026.

Dorris, M. C., Pare, M., \& Munoz, D. P. (1997). Neuronal activity in monkey superior colliculus related to the initiation of saccadic eye movements. Journal of Neuroscience, 17, $8566-8579$.

Dorris, M. C., Taylor, T. L., Klein, R. M., Munoz, D. P. (1999). Influence of previous visual stimulus or saccade on saccadic reaction times in monkey. Journal of Neurophysiology, 81, $2429-2436$.

Duhamel, J. R., Colby, C. L., \& Goldberg, M. E. (1992). The updating of the representation of visual space in parietal cortex by intended eye movements. Science, 255, 90-92.

Fries, W. (1984). Cortical projections to the superior colliculus in the macaque monkey: A retrograde study using horseradish peroxidase. Journal of Comparative Neurology, 230, 55-76.

Hikosaka, O., \& Wurtz, R. H. (1985). Modification of saccadic eye movements by GABA-related substances: II. Effects of muscimol in monkey substantia nigra pars reticulata. Journal of Neurophysiology, 53, 292-308.

Ivanoff, J., \& Klein, R. M. (2001). The presence of a nonresponding effector increases inhibition of return. Psychonomic Bulletin and Review, 8, 307-314.

Klein, R. M. (2000). Inhibition of return. Trends in Cognitive Sciences, 4, 138-147.

Klein, R. M., \& MacInnes, W. J. (1999). Inhibition of return is a foraging facilitator in visual search. Psychological Science, $10,346-352$.

Klein, R. M., Schmidt, W. C., \& Muller, H. J. (1998). Disinhibition of return: Unnecessary and unlikely. Perception and Psychophysics, 60, 862-872.

Maylor, E., \& Hockey, J. (1985). Inhibitory component of externally controlled covert orienting in visual space. Journal of Experimental Psychology: Human Perception and Performance, 11, 777-787.

McDonald, J. J., Ward, L. M., \& Kiehl, K. A. (1999). An event-related brain potential study of inhibition of return. Perception and Psychophysics, 61, 1411-1423.

Miller, E. K., Gochin, P. M., \& Gross, C. G. (1991). Habituationlike decrease in the responses of neurons in inferior temporal cortex of the macaque. Visual Neuroscience, 7 , $357-362$.

Moran, J., \& Desimone, R. (1985). Selective attention gates visual processing in the extrastriate cortex. Science, 229, $782-784$.

Munoz, D. P., Dorris, M. C., Pare, M., \& Everling, S. (2000). On your mark, get set: Brainstem circuitry underlying saccadic initiation. Canadian Journal of Physiology and Pharmacology, 78, 934-944.

Paré, M., \& Wurtz, R. H. (1997). Monkey posterior parietal cortex neurons antidromically activated from superior colliculus. Journal of Neurophysiology, 78, 3493-3497.

Perry, V. H., \& Cowey, A. (1984). Retinal ganglion cells that project to the superior colliculus and pretectum in the macaque monkey. Neuroscience, 84, 1125-1137.

Posner, M. I., \& Cohen, Y. (1984). Components of visual orienting. In H. Bouma \& D. G. Bouwhuis (Eds.), Attention and performance $X$ (pp. 531-556). Hillside, NJ: Erlbaum.

Posner, M. I., Rafal, R. D., Choate, L. S. \& Vaughan, J. (1985). Inhibition of return: Neural basis and function. Cognitive Neuropsychology, 2, 211-228.

Pratt, J., \& Castel, A. D. (2001). Responding to feature or location: A re-examination of inhibition of return and facilitation of return. Vision Research, 41, 3903-3908.

Rafal, R. D., Calabresi, P. A., Brennan, C. W., \& Sciolto, T. K. (1989). Saccade preparation inhibits reorienting to recently 
attended locations. Journal of Experimental Psychology:

Human Perception and Performance, 89, 673-685.

Reuter-Lorenz, P. A., Jha, A. P., \& Rosenquist, J. N. (1996). What is inhibited in inhibition of return? Journal of Experimental Psychology: Human Perception and Performance, 22, $367-378$.

Ro, T., \& Rafal, R. D. (1999). Components of reflexive visual orienting to moving objects. Perception and Psychophysics, 61, 826-836.

Robinson, D. L., Bowman, E. M., \& Kertzman, C. (1995). Covert orienting of attention in macaques: II. Contributions of parietal cortex. Journal of Neurophysiology, 95, 698-712.

Robinson, D. L., \& Kertzman, C. (1995). Covert orienting of attention in macaques: III. Contributions of the superior colliculus. Journal of Neurophysiology, 74, 713-721.

Sapir, A., Soroker, N., Berger, A., \& Henik, A. (1999). Inhibition of return in spatial attention: Direct evidence for collicular generation. Nature Neuroscience, 2, 1053-1054.

Schall, J. D. (1995). Neural basis of saccade target selection. Reviews of Neuroscience, 6, 63-85.

Simion, F., Valenza, E., Umilta, C., \& Dalla Barba, B. (1995). Inhibition of return in newborns is temporo-nasal asymmetrical. Infant Behavior and Development, 18, $189-194$

Snyder, L. H., Batista, A. P., \& Andersen, R. A. (1997). Coding of intention in the posterior parietal cortex. Nature, 386, $167-170$.

Snyder, J. J., \& Kingstone, A. (2000). Inhibition of return and visual search: How many separate loci are inhibited? Perception and Psychophysics, 62, 452-458.

Sparks, D. L., \& Hartwich-Young, R. (1989). The deep layers of the superior colliculus. In R. H. Wurtz \& M. E. Goldberg (Eds.), The neurobiology of saccadic eye movements, reviews of oculomotor research (vol. III, pp. 213-255). Amsterdam: Elsevier.

Stanford, T. R., Freedman, E. G., Sparks, D. L. (1996). Site and parameters of microstimulation: Evidence for independent effects on the properties of saccades evoked from the primate superior colliculus. Journal of Neurophysiology, 76, 3360-3381.
Steinmetz, M. A., Connor, C. E., Constantinidis, C., \& McLaughlin, J. R. (1994). Covert attention suppresses neuronal responses in area $7 \mathrm{a}$ of the posterior parietal cortex. Journal of Neurophysiology, 72, 1020-1023.

Steinmetz, M. A., \& Constantinidis, C. (1995). Neurophysiological evidence for a role of posterior parietal cortex in redirecting visual attention. Cerebral Cortex, 5, $448-456$.

Stuphorn, V., Hoffmann, K. P., \& Miller, L. E. (1999). Correlation of primate superior colliculus and reticular formation discharge with proximal limb muscle activity. Journal of Neurophysiology, 81, 1978-1982.

Tassinari, G., \& Berlucchi, G. (1993). Sensory and attentional components of slowing of manual reaction time to non-fixated visual targets by ipsilateral primes. Vision Research, 93, 1525-1534.

Taylor, T. L., \& Klein, R. M. (1998). On the causes and effects of inhibition of return. Psychonomic Bulletin and Review, 5, 625-643.

Taylor, T. L., \& Klein, R. M. (2000). Visual and motor effects in inhibition of return. Journal of Experimental Psychology: Human Perception and Performance, 26, $1639-1656$.

Tipper, S. P., Rafal, R. D., Reuter-Lorenz, P., Staarveldt, Y., Ro, T., Egly, R., Danziger, S., \& Weaver, B. (1997). Objectbased facilitation and inhibition from visual orienting in the human split-brain. Journal of Experimental Psychology: Human Perception and Performance, 23, $1522-1532$.

Tipper, S. P., Weaver, B., Jerreat, L. M., Burak, A. L. (1994). Object-based and environment-based inhibition of return of visual attention. Journal of Experimental Psychology: Human Perception and Performance, 20, 478-499.

Weaver, B., Lupianez, J., \& Watson, F. L. (1998). The effects of practice on object-based, location-based, and staticdisplay inhibition of return. Perception and Psychophysics, 60, 993-1003.

Wurtz, R. H., \& Mohler, C. W. (1976). Organization of monkey superior colliculus: Enhanced visual response of superficial layer cells. Journal of Neurophysiology, 39, 745-765. 